Cerebrovasc Dis 2010;29:412

DOI: 10.1159/000288057

\section{Symmetrical Cerebral Arteriovenous Malformations in Bilateral Basal Ganglia}

Kazuki Nagayama ${ }^{\text {a, }}$, Hiroki Kurita ${ }^{\text {b, }}$

a Department of Neurosurgery, Himon'ya Hospital, and

${ }^{b}$ Department of Neurosurgery, Kyorin University Faculty of

Medicine, Tokyo, and ' Department of Cerebrovascular Surgery,

International Medical Center, Saitama Medical University,

Saitama, Japan

A 23-year-old otherwise healthy woman developed left hemiparesis because of small right thalamic hemorrhage. The cerebral angiography revealed symmetrical arteriovenous malformations (AVMs) in the bilateral basal ganglia. Each AVM was supplied by ipsilateral lenticulostriate and choroidal arteries, which drained into the galenic venous system (fig. 1). The patient recovered well and the AVM was subsequently treated with focused irradiation therapy.

Multiple cerebral AVMs are very rare. The present case is unique in its symmetrical strategic localization and serves as a reminder of the clinicoradiological variability displayed by an AVM.
Kazuki Nagayama, MD, PhD

Department of Neurosurgery

Himon'ya Hospital, 2-9-7, Minami

Meguro-ku, Tokyo 152-0013 (Japan)

Tel. +8133723 1515, Fax +81337231816

E-Mail knagayama-nsu@umin.ac.jp
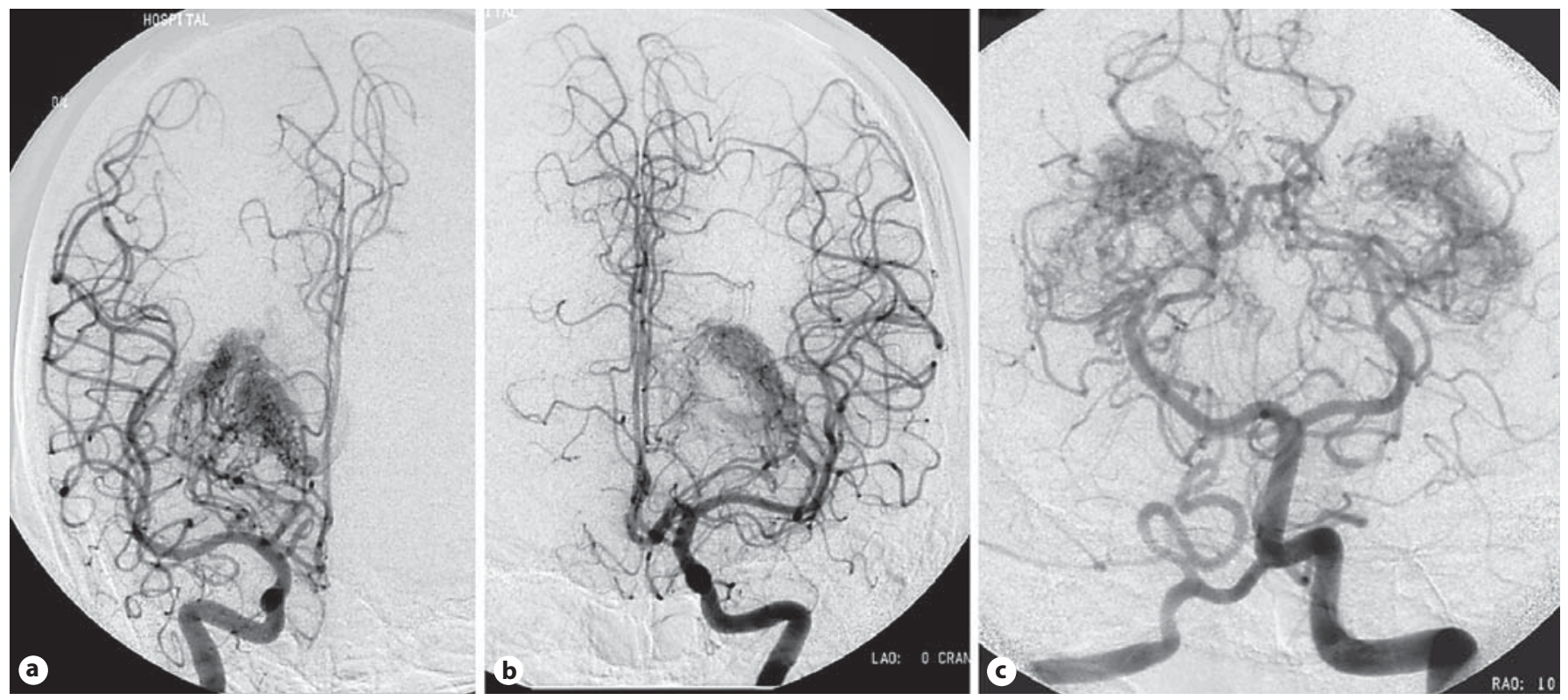

Fig. 1. Carotid angiograms - right side (a) and left side (b) - and left vertebral angiograms of the arterial phase (c), showing symmetrical bilateral basal ganglion AVMs. 